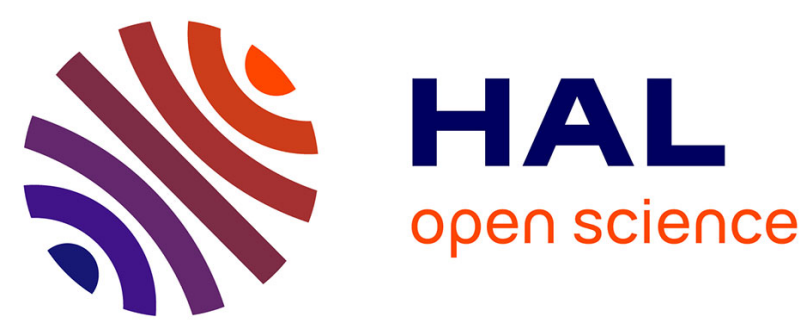

\title{
Editorial : Bisubstrate inhibitors: the promise of a selective and potent chemical inhibition of epigenetic "writers"
}

Corentin Bon, Ludovic Halby, Paola B Arimondo, Paola B Arimondo

\section{- To cite this version:}

Corentin Bon, Ludovic Halby, Paola B Arimondo, Paola B Arimondo. Editorial: Bisubstrate inhibitors: the promise of a selective and potent chemical inhibition of epigenetic "writers". Epigenomics, 2020, 12 (17), pp.1479-1482. 10.2217/epi-2020-0203 . pasteur-03023370

\section{HAL Id: pasteur-03023370}

https://hal-pasteur.archives-ouvertes.fr/pasteur-03023370

Submitted on 23 Nov 2021

HAL is a multi-disciplinary open access archive for the deposit and dissemination of scientific research documents, whether they are published or not. The documents may come from teaching and research institutions in France or abroad, or from public or private research centers.
L'archive ouverte pluridisciplinaire HAL, est destinée au dépôt et à la diffusion de documents scientifiques de niveau recherche, publiés ou non, émanant des établissements d'enseignement et de recherche français ou étrangers, des laboratoires publics ou privés. 


\title{
Bisubstrate inhibitors: the promise of a selective and potent chemical inhibition of epigenetic "writers"
}

\author{
Corentin Bon, Ludovic Halby, Paola B. Arimondo
}

EpiCBio, Epigenetic Chemical Biology, Department Structural Biology and Chemistry, Institut Pasteur,

CNRS UMR n³523, 28 rue du Dr Roux, 75015 Paris, France

Corresponding author: Paola B. Arimondo, paola.arimondo@cnrs.fr

Keywords: methyltransferases, bisubstrate inhibitors, SAM analogues, substrate analogues, histone, DNA, HMT, DNMT, epigenetics.

On January 23, 2020, the Food and Drug Administration approved tazemetostat (TAZVERIK), developed by Epizyme Inc., for patients aged sixteen years and older with metastatic or locally advanced epithelioid sarcoma not eligible for complete resection. It is the first inhibitor of histone methyltransferase (HMT) to be approved. Histone methylation, as other epigenetic mechanisms, is involved in gene expression without changing the underlying DNA sequence. Abnormalities in the epigenetic profile are implicated in various diseases (such as cancer, infectious, autoimmune and neurodegenerative diseases). Transferases are major actors in epigenetic modulations chemically modifying histones and DNA. The Tazemetostat's success story is based on the understanding of the biology of the target protein in certain cancers and a lead optimization process from a high-throughput screening's hit. The biological target is the HMT Enhancer of zeste homolog 2 (EZH2). It is the enzymatic subunit of the Repressive Complex Polycomb 2 (PRC2) that methylates lysine 27 of histone 3 (H3K27), promoting transcription repression. Several cancers are associated with hypermethylation of H3K27, which are due either to a hyperactivity of EZH2 mutants or to a loss of activity of the corresponding demethylases, Ubiquitously-transcribed X chromosome tetratricopeptide repeat protein (UTX). Structure Activity Relationship (SAR) studies of the hit molecule allowed the discovery of EPZ6438, a nanomolar inhibitor of EZH2. EPZ6438 acts as a competitor of the co-factor $S$-adenosine-L-methionine (SAM)[1], the donor of the methyl group transferred by the enzyme to the lysine, and thus inhibits the methylation of H3K27. The compound showed an extraordinary selectivity against EZH2. As all methyltransferases use SAM as co-factor, it is a challenge to design selective inhibitors that compete with the SAM. Indeed, several other SAM analogues have been synthesised to target HMT, but the selectivity and potency were not sufficient to reach clinical trial[2].

Importantly, all transferases, such as HMT, DNA methyltransferases (DNMT) and histone acetyltransferases (HAT), bear two pockets: a co-factor binding site and a pocket that binds the substrate to be modified. Chemical strategies are developed to inhibit these enzymes by targeting either the co-factor binding site, the substrate pocket or both at the same time. Single pocket targeting such as the case of Tazemetostat is often not sufficient for selectivity, whereas the targeting of both pockets potentially leads to more potent and selective compounds. 
The epigenetic methyltransferases (MTases) HMT and DNMT have over 30 members, which all share the same co-factor to methylate three different substrates (lysine for KMT, arginine for PRMTs and cytosine for DNMT). The discovery of selective inhibitors is highly challenging. The SAM pocket differs among the families of MTases to accommodate the respective substrate and co-factor, orienting the methyl group adaptively to the substrate. Indeed, the orientation of the SAM is different in PRMTs compared to KMTs containing the SET-domain, and even further in DNMTs[3]. Consequently, it is possible to obtain selectivity for one family versus another by imposing a particular bending of the SAM competitor, but further selectivity in the family is made difficult by the high similarity in the binding mode of the SAM[4].

However, there are variations of the amino acid sequence of the substrate pocket from one protein to another, and it is thus possible to design small molecules that compete with the substrate and take advantage of the level of methylation, the position in the histone tail or the 3D conformation of the substrate. For example, KMTs methylate in a highly selective way only one specific lysine in the histone tail and can achieve mono-, di- and/or tri-methylation depending on the chromatin state. Both Euchromatic histone-lysine N-methyltransferase 2 (EHMT2), also known as G9A, and suppressor of variegation 3-9 (SUV39) methylate H3K9 but not in the same chromatin state [5], while the PRMTs achieve either symmetric or asymmetric methylation of arginine. In the case of the DNMTs, potential selectivity could be achieved based on either the small differences in amino acids of the catalytic pocket[6] or some sequence specificity of the DNA sequence around the CpG site[7],[8].

The most promising strategy for selective inhibitors resides in targeting both the cofactor and substrate pockets at the same time. This strategy is based on the design of bisubstrate derivatives. Bisubstrate derivatives consists of analogues of the co-factor and analogues of the substrate covalently linked together. A notable case is the transition state analogues (TSA) that consist in the covalent coupling of the substrate and the co-factor exactly. It was the first strategy that led to the discovery of selective inhibitors of kinase proteins[9]. In addition to selectivity, it bears the promise of potency because the free binding energy of the formed conjugate is equal to the sum of the free binding energies of the moieties plus an entropy gain due to the interaction of a single molecule rather than multiple fragments within the protein. Of note, part of the binding energy is generally lost during the conjugation of the components, due to the fact that they lose freedom of orientation in their respective pocket. However, linear decrease in the free bond energy causes an exponential decrease in the dissociation constant and the entropic effect can be up to three order fold increase[9].

Notably, in the design of bisubstrate analogues, the linker that binds the two moieties plays an important role; its nature and length need to be optimised in parallel to the analogues of substrate and co-factor. The linker's optimization depends on the distance between the two pockets and the 3D orientation of the substrate relative to the co-factor. The length dictates how each moiety fits in its respective pocket, while on the nature of the linker will depend its capability to interact or repulse with the interspace-forming amino acids. For example in DNMT1, it has been suggested that a positively charged linker should allow better potency[10]. Moreover, a preorganization of the moieties using a constrained linker could allow the two parts to be correctly directed toward their respective pockets, optimising the entropy gain. Thus, the linker design is a key point in the modulation for selectivity: it can allow us to optimise the interaction with one enzyme and destabilize the conformation for the others.

There are several examples of success of the bisubstrate strategy targeting the epigenetic MTases. We developed bisubstrate inhibitors of DNMT using aminoquinazoline as an adenosine mimic of the SAM. Various linkers and second substrates were evaluated leading to potent inhibitors of two isoforms of DNMT[11]. Similarly, a selective PRMT4 inhibitor was 
discovered using an adenosine derivative coupled to a cytosine derivative as second substrate [12].

Two representative examples of bisubstrates that reached clinical trial are EPZ5676 and JNJ-64619178, targeting DOT1L and PRMT5, respectively. DOT1L is a KMT that is involved in the proliferation of MLL rearranged leukaemia cells[9]. Successive modulations of the three parts of the bisubstrate strategy (co-factor, linker, substrate) resulted in a potent and highly selective DOT1L inhibitor[9]. Epizyme, Inc. minimized the SAM co-factor keeping the recognition pattern of the molecule, while reducing the polarity. Further modifications showed the necessity of a protonated tertiary amine on the linker and the addition of a hydrophobic moiety for activity and selectivity, leading to a first potent bisubstrate inhibitor EPZ4777. By constraining the linker, Epizyme, Inc. obtained the optimised EPZ5676. Most interestingly, the binding mode is most efficient resulting from a change in conformation of the protein upon binding, leading to the occupancy of the SAM pocket and a closure of the substrate pocket by formation of a new hydrophobic pocket adapted to the compound. New bisubstrate-type inhibitors have now been designed by Novartis to obtain more stable, potent and orally available molecules[14]. PRMT5 (Arginine protein methyltransferase 5) is an enzyme responsible for the symmetric di-methylation of arginine and a therapeutic target in many cancers, as glioblastoma, leukaemia, lymphoma, prostate cancer or colorectal cancer[15]. Two inhibitors have been developed and are in phase I of clinical trials. GSK3326595 occupies only the substrate pocket via a tetrahydro-isoquinoline-type structure making cation- $\pi$ type interactions and is a reversible inhibitor with a fairly good selectivity[15]. JNJ-64619178 binds simultaneously to the SAM and protein substrate-binding pockets of the PRMT5. It presents high selectivity and a rapid pseudo-irreversible mode-of-action, showing a potent and broad inhibition of cancer cellular growth[16]. To date, this molecule is the most potent and selective compound developed against PRMT5, showing the efficiency of this strategy[17].

In conclusion, bisusbtrate inhibitors are the most promising for potent and selective inhibition of epigenetic writers. The strategy has the advantage of involving the interaction between two components (the protein and the compound) instead of three (the protein, the substrate and the co-factor). Thanks to this strategy, potent and selective inhibitors were also obtained for acetyltransferases[18], in addition to DNMTs[11], DOT1L[9] and PRMT5[16]. New bisubstrate analogues are to be explored to target other epigenetic writers. For the design of bisubstrate analogues it is necessary to modulate the three items (co-factor, substrate and linker), which can be challenging. An interesting option is to optimize each moiety through SAR or virtual screening for each pocket and then couple the two fragments by various linkers. Another approach is the in situ chemical coupling of substrate and co-factor analogues, in which the success of the chemical coupling is guided by the target protein[19]. In all cases, bisubstrate inhibitors, a classical medicinal chemistry approach, constitute an efficient strategy to obtain selective and potent probes for the writers.

\section{Bibliography:}

[1] "Selective Inhibition of EZH2 by EPZ-6438 Leads to Potent Antitumor Activity in EZH2Mutant Non-Hodgkin Lymphoma | Molecular Cancer Therapeutics." https://mct.aacrjournals.org/content/13/4/842.long (accessed Apr. 16, 2020).

[2] J. Zhang and Y. G. Zheng, "SAM/SAH Analogs as Versatile Tools for SAM-

Dependent Methyltransferases,” ACS Chem. Biol., vol. 11, no. 3, pp. 583-597, Mar. 2016, doi: 10.1021/acschembio.5b00812.

[3] V. Campagna-Slater, M. W. Mok, K. T. Nguyen, M. Feher, R. Najmanovich, and M. Schapira, "Structural chemistry of the histone methyltransferases cofactor binding site," $J$. 
Chem. Inf. Model., vol. 51, no. 3, pp. 612-623, Mar. 2011, doi: 10.1021/ci100479z.

[4] C. Qian and M.-M. Zhou, "SET domain protein lysine methyltransferases: Structure, specificity and catalysis," Cell. Mol. Life Sci. CMLS, vol. 63, no. 23, pp. 2755-2763, Dec. 2006, doi: 10.1007/s00018-006-6274-5.

[5] H. Wu et al., "Structural Biology of Human H3K9 Methyltransferases," PLoS ONE, vol. 5, no. 1, Jan. 2010, doi: 10.1371/journal.pone.0008570.

[6] A. Erdmann, L. Halby, J. Fahy, and P. B. Arimondo, "Targeting DNA Methylation with Small Molecules: What's Next?," J. Med. Chem., vol. 58, no. 6, pp. 2569-2583, Mar. 2015, doi: 10.1021/jm500843d.

[7] V. Handa and A. Jeltsch, "Profound flanking sequence preference of Dnmt3a and Dnmt3b mammalian DNA methyltransferases shape the human epigenome," J. Mol. Biol., vol. 348, no. 5, pp. 1103-1112, May 2005, doi: 10.1016/j.jmb.2005.02.044.

[8] M. Emperle et al., "The DNMT3A R882H mutant displays altered flanking sequence preferences," Nucleic Acids Res., vol. 46, no. 6, pp. 3130-3139, Apr. 2018, doi: 10.1093/nar/gky168.

[9] D. Lavogina, E. Enkvist, and A. Uri, "Bisubstrate Inhibitors of Protein Kinases: from Principle to Practical Applications," ChemMedChem, vol. 5, no. 1, pp. 23-34, Jan. 2010, doi: 10.1002/cmdc.200900252.

[10] V. Miletić, I. Odorčić, P. Nikolić, and Ž. M. Svedružić, "In silico design of the first DNA-independent mechanism-based inhibitor of mammalian DNA methyltransferase Dnmt1," PloS One, vol. 12, no. 4, p. e0174410, 2017, doi: 10.1371/journal.pone.0174410.

[11] "Rational Design of Bisubstrate-Type Analogues as Inhibitors of DNA Methyltransferases in Cancer Cells. - PubMed - NCBI."

https://www.ncbi.nlm.nih.gov/pubmed/28463515 (accessed Apr. 17, 2020).

[12] L. Halby et al., "Hijacking DNA methyltransferase transition state analogues to produce chemical scaffolds for PRMT inhibitors," Philos. Trans. R. Soc. Lond. B. Biol. Sci., vol. 373, no. 1748, 05 2018, doi: 10.1098/rstb.2017.0072.

[13] "Conformational Adaptation Drives Potent, Selective and Durable Inhibition of the Human Protein Methyltransferase DOT1L - Basavapathruni - 2012 - Chemical Biology \&amp; Drug Design - Wiley Online Library."

https://onlinelibrary.wiley.com/doi/full/10.1111/cbdd.12050 (accessed Apr. 17, 2020).

[14] C. Chen et al., "Discovery of Novel Dot1L Inhibitors through a Structure-Based Fragmentation Approach," ACS Med. Chem. Lett., vol. 7, no. 8, pp. 735-740, Jun. 2016, doi: 10.1021/acsmedchemlett.6b00167.

[15] S. V. Gerhart et al., "Activation of the p53-MDM4 regulatory axis defines the antitumour response to PRMT5 inhibition through its role in regulating cellular splicing," Sci. Rep., vol. 8, no. 1, p. 9711, 26 2018, doi: 10.1038/s41598-018-28002-y.

[16] D. Brehmer et al., "Abstract DDT02-04: A novel PRMT5 inhibitor with potent in vitro and in vivo activity in preclinical lung cancer models," Cancer Res., vol. 77, no. 13 
Supplement, pp. DDT02-04-DDT02-04, Jul. 2017, doi: 10.1158/1538-7445.AM2017DDT02-04.

[17] H. Tao, X. Yan, K. Zhu, and H. Zhang, "Discovery of Novel PRMT5 Inhibitors by Virtual Screening and Biological Evaluations," Chem. Pharm. Bull. (Tokyo), vol. 67, no. 4, pp. 382-388, Apr. 2019, doi: 10.1248/cpb.c18-00980.

[18] L. Ngo, T. Brown, and Y. G. Zheng, "Bisubstrate inhibitors to target histone acetyltransferase 1," Chem. Biol. Drug Des., vol. 93, no. 5, pp. 865-873, 2019, doi: 10.1111/cbdd.13476.

[19] T. Osborne, R. L. W. Roska, S. R. Rajski, and P. R. Thompson, "In situ generation of a bisubstrate analogue for protein arginine methyltransferase 1," J. Am. Chem. Soc., vol. 130, no. 14 , pp. 4574-4575, Apr. 2008, doi: $10.1021 / \mathrm{ja} 077104 \mathrm{v}$. 